\title{
Modal analysis for implant stability assessment: sensitivity of this methodology for different implant designs
}

DOI:

10.1016/j.dental.2018.05.016

\section{Document Version}

Accepted author manuscript

Link to publication record in Manchester Research Explorer

\section{Citation for published version (APA):}

Zanetti, E. M., Ciaramella, S., CALî, M., Pascoletti, G., Martorelli, M., Asero, R., \& WATTS, D. C. (2018). Modal analysis for implant stability assessment: sensitivity of this methodology for different implant designs. Dental Materials, 34(8), 1235-1245. https://doi.org/10.1016/j.dental.2018.05.016

\section{Published in:}

Dental Materials

\section{Citing this paper}

Please note that where the full-text provided on Manchester Research Explorer is the Author Accepted Manuscript or Proof version this may differ from the final Published version. If citing, it is advised that you check and use the publisher's definitive version.

\section{General rights}

Copyright and moral rights for the publications made accessible in the Research Explorer are retained by the authors and/or other copyright owners and it is a condition of accessing publications that users recognise and abide by the legal requirements associated with these rights.

\section{Takedown policy}

If you believe that this document breaches copyright please refer to the University of Manchester's Takedown Procedures [http://man.ac.uk/04Y6Bo] or contact uml.scholarlycommunications@manchester.ac.uk providing relevant details, so we can investigate your claim.

\section{OPEN ACCESS}




\section{Modal analysis for implant stability assessment: sensitivity of this methodology for different implant designs}

Elisabetta Maria ZANETTI $^{1}$, Stefano CIARAMELLA ${ }^{2 *}$, Michele CALì ${ }^{3}$, Giulia PASCOLETTI $^{1}$, Massimo MARTORELLI ${ }^{2}$, Riccardo ASERO ${ }^{4}$ and David C WATTS ${ }^{5}$

${ }^{1}$ Department of Engineering - University of Perugia, Via Duranti 67, 06125 Perugia, Italy. elisabetta.zanetti@unipg.it

${ }^{2}$ Department of Industrial Engineering, Fraunhofer JL IDEAS - University of Naples Federico II, P.le Tecchio, 80 - 80125 Napoli, Italy. ing.stefano.ciaramella@gmail.com

${ }^{3}$ Electric, Electronics and Computer Engineering Department, University of Catania, V.le A. Doria, 6 95125 Catania, Italy. mcali@dii.unict.it

${ }^{4}$ Studio Odontoiatrico Asero, Via Generale Cantore 23, 95123 Catania, Italy. riccardoasero@me.com

${ }^{5}$ School of Medical Sciences and Photon Science Institute, University of Manchester, United Kingdom. david.watts@manchester.ac.uk

* Corresponding author: ing.stefano.ciaramella@gmail.com 
Keywords: Reverse Engineering; CAD; Finite Element Analysis; Dental Materials; Material Properties; Implant Stability; Bone Properties; Endosteal Implants; Osseointegration

\begin{abstract}
Objective: To investigate the influence of implant design on the change in the natural frequency of boneimplant system during osseointegration by means of a modal 3D Finite Element Analysis.

Methods: Six implants were considered. Solid models were obtained by means of reverse engineering techniques. The mandibular bone geometry was built-up from a CT scan dataset through image segmentation. Each implant was virtually implanted in the mandibular bone. Two different models have been considered, differing in the free length of the mandibular branch ('long branch' and 'short branch') in order to simulate the variability of boundary conditions when performing vibrometric analyses. Modal analyses were carried out for each model, and the first three resonance frequencies were assessed with the respective vibration modes.
\end{abstract}

Results: with reference to the 'long branch' model, the first three modes of vibration are whole bone vibration with minimum displacement of the implant relative to bone, with the exception of the initial condition ( $1 \%$ bone maturation) where the implant is not osseointegrated. By contrast, implant displacements become relevant in the 'short branch' model, unless osseointegration level is beyond $20 \%$. The difference between resonance frequency at whole bone maturation and resonance frequency at $1 \%$ bone maturation remained lower than $6.5 \%$ for all modes, with the exception of the third mode of vibration in the ' $\mathrm{D}$ ' implant where this difference reached $9.7 \%$. With reference to the 'short branch', considering the first mode of vibration, $61-68 \%$ of the frequency increase was achieved at $10 \%$ osseointegration; $72-79 \%$ was achieved at $20 \% ; 89-93 \%$ was achieved at $50 \%$ osseointegration. The pattern of the natural frequency versus the osseointegration level is similar among different modes of vibration.

Significance: Resonance frequencies and their trends towards osseointegration level may differ between implant designs, and in different boundary conditions that are related to implant position inside the mandible; tapered implants are the most sensitive to bone maturation levels, small implants have very little sensitivity. Resonance frequencies are less sensitive to bone maturation level beyond 50\%. 


\section{Introduction}

As it is well known, implant stability plays a major role in successful dental implantology. Primary stability (a mechanical phenomenon) is achieved when an implant has just been set in place and is related to bone quality [1], implant geometry [2][3], and to the surgical technique [4]. Secondary stability (a biological phenomenon) is achieved via implant osseointegration, where new bone formation leads to an increase in stiffness of the peri-implant bone, and to bone-implant interlocking.

Optimizing secondary stability requires limiting micro-movements between the implant and the bone below $100 \mu \mathrm{m}$ [5], since they can potentially lead to fibrous bone formation [6]. Therefore a healing period, during which the implants are unloaded, is required.

On the other hand, load application to an implant is necessary to provide stimulus for bone maturation [6]. Consequently, improved implant designs and surface treatments have led to new loading protocols, such as 'early loading' or 'immediate loading' [7].

The question is therefore: how long should the healing period last? This debate is still open and is the object of many research studies [8,9]. Seemingly, absolute indications cannot be given since the patient bone quality, the kind of implant and the outcome of surgical technique play a fundamental role $[7,10,11]$.

Establishing patient-specific loading protocols requires a non-invasive quantitative assessment of implant stability. An objective, quantitative measurement of implant stability prior to loading is very desirable, even if patients self-regulate masticatory load levels in relation to implant stability, to a certain extent [12].

Vibrometry techniques could realize this aim, according to extensive data reported in the literature where numerical, experimental and clinical methods have been employed to establish the relationship between resonance frequencies and cortical bone thickness [13,14], trabecular bone density [13,15-19], implant length [14,20,21], implant diameter [22,23] and time elapsed since implant placement [18,21,24].

Clinical studies have also been performed, to gain quantitative data about the lower limit of resonance frequency providing optimal primary stability $[21,25,26]$, or the required frequency to start implant loading [25].

However, it is not clear if, and to what extent, the sensitivity of this technique, i.e. resonance frequency variation during osseointegration, is affected by implant design. Very different implant shapes are now available (long or short; cylindrical or tapered), with different threads (single or double thread; short or large 
pitch, etc), and the most sensitive vibration mode - the mode undergoing the highest frequency variation during osseointegration - might differ amongst implant designs.

The aim of the present study was to assess the influence of implant design on the resonance frequency variation during osseointegration, using Resonance Frequency Analysis (RFA). Details concerning the experimental set up and the boundary conditions of bone were also investigated, since they can have a considerable influence on the sensitivity of resonance frequencies to the osseointegration level: under specific circumstances the osseointegration effect could become completely uninfluential, as detailed in the following section.

\section{Materials and Methods}

Modern CAD-FEM (Computer Aided Design and Finite Element Method) methodologies have been extensively used in biomedical investigations to characterize biomechanical responses in dental applications $[27-32]$.

Starting from these methodologies, four implants from the same manufacturer (ISOMED ${ }^{\circledR}$ Dental Implant, Italy) were considered and analyzed to investigate the influence of implant geometrical factors, such as shape and thread pitch, on the change of the natural frequency (i.e. resonance frequency) of bone-implant system during osseointegration.

These implants were designated as: A (cylindrical implant with internal hexagon connection TIC-10); B (conical implant with inside hexagon connection and medium thread TICc-10 BL); C (conical transmucous implant with internal hexagon connection and double thread TVI5-3-Tr-PLUS) and D (conical implant with internal hexagon connection and progressive thread PROGRESSIVE 5-10). The geometrical features of these implants are shown in Fig. 1.

\subsection{Generation of solid models}

Solid models of ISOMED ${ }^{\circledR}$ Dental implants were obtained by means of reverse engineering techniques [11]: the outer shape was digitised by a laser scanner: CAM2 Edge SCANARM HD - FARO (accuracy $\pm 25 \mu \mathrm{m}$ ). Point clouds were imported into Geomagic Studio ${ }^{\circledR}$ software, where 3D tessellated surfaces were created, and sharp edges and cross section curves were obtained through feature detection algorithms [33]. Finally, 
the implant parametric 3D CAD model was created using SolidWorks ${ }^{\circledR}$ software ver. 2017 (SolidWorks Corporation, Concord, MA, USA).

The mandibular bone geometry was established using CT scan images (slice thickness $1 \mathrm{~mm}$, standard energy and current levels: $120 \mathrm{kV}$ and $200 \mathrm{~mA}$ ). Image data sets were processed using Mimics ${ }^{\circledR}$ software by Materialise. CAD 3D volume reconstruction was performed using Rhinoceros ${ }^{\odot}$ software by Robert McNeel \& Associates. The geometry was subsequently sectioned to focus the analysis on the portion of the mandibular branch containing the implant (Fig. 2).

Each implant was virtually placed in the mandibular bone volume. Boolean operations were carried out to ensure the congruence of interfacial boundaries of the implant and bone.

The transverse mandibular section, shown in Fig. 2, consists of four distinct areas: an outer shell of cortical bone with $1.2 \mathrm{~mm}$ thickness in the coronal area, an inner volume of trabecular bone and two interfacial volumes. The interfacial volumes extend up to $1 \mathrm{~mm}$ external to the implant thread [22] and represent the cortical and trabecular bone regions, respectively. They are affected by implant placement and their properties will change, as a result of progressive implant osseointegration [19].

Implant models were placed in a coordinate system, where the 1-axis and 2-axis were chosen for the buccolingual direction and mesio-distal direction, respectively, while the 3-axis was oriented upwards (Fig. 2a).

\subsection{Numerical simulation}

The effect of the level of osseointegration on the natural frequencies of implant vibration was analyzed by means of a modal three-dimensional Finite Element (FE) Analysis using ANSYS $^{\circledR}$ v. 17.0.

In total, six different FE models were created and analyzed. ANSYS software was used to mesh boneimplant system components. All volumes were discretized by 10-node tetrahedral elements TET10 with a global size ranging from $6.0 \cdot 10^{-5} \mathrm{~mm}$ to $0.5 \mathrm{~mm}$. The total number of elements was equal to about 190,000 with approximately 320,000 nodes. To minimize the mesh-dependency of results, due to small radius of curvature and notch effects, mesh refinement techniques were used.

Mechanical properties assigned to each material are reported in Table 1, where the $k$ parameter was used to simulate the variation of bone mechanical properties during osseointegration. For example, $k=0.5$ when the bone, during its maturation, has reached $50 \%$ of its mechanical properties at full maturation. For the density 
at both trabecular and cortical bone interface volumes, the $k^{1 / 3}$ parameter was used, according to [34]. Both cortical and trabecular bones were modelled as transversely isotropic and fully characterized by five independent elastic constants: $E_{1}=E_{3}, E_{2}, v_{12}=v_{23}, v_{13}, G_{12}=G_{23}$, and the direction of the main orthotropy axes, as deduced from the literature [2].

The mandibular branch was fully constrained in correspondence with its extreme sections; two different lengths $(15 \mathrm{~mm}$ and $30 \mathrm{~mm})$ of mandibular branch portion have been considered: the first length reproduces a geometry commonly studied in numerical analyses of implant modes of vibration [13,19]; the second length has been studied in order to check how modes of vibration would change in relation to free bone length that is in relation to different implant positions along the mandible or different mandible constrains (tight/slack muscles, supported/unsupported chin) .

A modal analysis was carried out for each boundary condition, and the first three resonance frequencies were evaluated with the respective vibration modes.

The resonance frequencies represent natural vibration frequencies of a structure when it is moved from its stationary conditions (like a plucked string). They are related, as are all such mechanical systems [35], to structural stiffness, mass distribution, and boundary conditions. Finite element programs are able to calculate the value of resonance frequencies provided that the stiffness matrix, mass matrix and boundary conditions are defined. Structures can have more than one mode of vibration, corresponding to different deformed shapes. For example, a truss can bend on two planes or it can undergo torsion; consequently more than one resonance frequencies are calculated, and they are usually sorted in an ascending order.

\section{Results}

In this section, the change in the natural frequency versus osseointegration level, for different implant designs, was reported and analyzed.

Fig. 3 depicts, for implants A, B, C and D, the first three modal frequency trends for the first boundary condition case ('long branch'). All curves have a similar pattern: the resonance frequency sharply increases, corresponding to a bone maturation level between $1 \%$ and $10 \%$. But for bone maturation levels greater than $10 \%$, the sensitivity of the resonance frequency to bone maturation reduced. Fig. 4 shows modal shapes for $\mathrm{k}>0.01$ : the first vibration mode was due to bone flexion about the ' 3 ' axis; the second vibration mode was 
produced by bone flexion about the ' 1 ' axis, and, finally, the third vibration mode was due to bone torsion. Only for $\mathrm{k}=0.01$ was the implant displacement inside the bone evident. With reference to the first mode of vibration - and considering the frequency difference between $100 \%$ and $1 \%$ integration as a reference - over $45 \%$ of this increase was achieved at $10 \%$ osseointegration; $64-73 \%$ was achieved at $20 \%$; $85-89 \%$ was achieved at $50 \%$ osseointegration. However, the difference between the resonance frequency at whole bone maturation and the resonance frequency at $1 \%$ bone maturation remained less than $6.9 \%$ for all modes, with the exception of the third mode of vibration in 'implant D' where this difference reached $9.7 \%$. Implant 'E' produced a significant difference only in relation to the second mode of vibration $(2.7 \%)$, since osseointegration resulted in a $0.5 \%$ and $0.8 \%$ variation for the first and third mode of vibration, respectively. This implant exhibited the smallest variations in resonance frequencies due to its small size. Differences in resonance frequency between models ' $\mathrm{E}$ ' and ' $\mathrm{D}$ ' reached $10.5 \%$ (for the second mode) and were equal to $7.4 \%$ for the first mode.

Further evaluation was made for a shorter mandibular branch ('short branch'), as detailed in the previous paragraph, with the aim of comparing results with previous research, reducing mandibular bone deformation and giving more emphasis to implant displacement within the bone.

The pattern of frequency increases versus osseointegration level (Fig. 5) was still non-linear, but more gradual than in Fig. 3. The modes of vibration were completely different, since implant displacements within the bone now played a greater role (Fig. 6). In more detail: the first modal shape was still due to bone flexion about the ' 3 ' axis; the second was a vertical displacement of the implant inside bone (here the implant behaves as if it were constrained with slide contact at the interface volume of trabecular bone [36]) for low osseointegration levels, while this same mode of vibration was due to bone flexion about ' 1 ' axis for high $(>20 \%)$ osseointegration levels. Finally, the third modal shape was due to a rotation of the implant about the ' 2 ' axis at low osseointegration levels and to bone torsion at high osseointegration levels ( $>20 \%)$.

With reference to the first mode of vibration - and considering the frequency difference between $100 \%$ and $1 \%$ integration as a reference - $61-68 \%$ of this increase was achieved at $10 \%$ osseointegration; $72-79 \%$ was achieved at $20 \% ; 89-93 \%$ was achieved at $50 \%$ osseointegration. The difference between resonance frequency at whole bone maturation and resonance frequency at $1 \%$ bone maturation remained less than $7.4 \%$ for the first mode of vibration, but it reached over $16.8 \%$ for the second and third modes; the second 
mode of vibration being slightly more sensitive. However, implant 'E' underwent variation of only 5.6,] and 9.2\%. In more detail, the implant ' $\mathrm{D}$ ' model allowed the most sensitive estimate of osseointegration level from the resonance frequency for the first mode of vibration $(7.4 \%$ difference when moving from $1 \%$ to $100 \%$ osseointegration level). The ' $\mathrm{C}$ ' implant, for the second and third modes of vibration, exhibited $16.9 \%$ and $16.2 \%$ differences, when moving from $1 \%$ to $100 \%$ osseointegration level, respectively). Differences in resonance frequency between models ' $\mathrm{E}$ ' and ' $\mathrm{D}$ ' reached $20.0 \%$ (for the second mode) and were equal to $14.5 \%$ for the first mode.

The first mode is the reference mode for usual types of implant device [21].

\section{Discussion}

The bone implant system can be analysed as a vibrating mechanical system, made of two components: the mandibular bone and the implant. Unless the mandibular bone is sufficiently secured, vibration will be largely dominated by the low-frequency, global flexural modes of the bone block which are independent of the level of osseointegration. If the mandibular bone is sufficiently secured, then the effect of osseointegration on the natural frequency can be predicted with reference to a classical dynamic system made of a mass, suspended on a spring, where the lumped mass is the implant (Fig. 7b). The complexity of the system is due to the stiffness being different along the implant length. In clinical reality, the whole implant is surrounded by a maturing bone cylinder $\left(\mathrm{K}_{1}\right.$ in Fig. $\left.7 \mathrm{~b}\right)$. This cylinder is bounded by a cortical bone shell cervically ( $\mathrm{K}_{2}$ in Fig. $7 \mathrm{~b}$ ), while it is bounded by a trabecular bone volume apically $\left(\mathrm{K}_{3}\right.$ in Fig. $7 b$ ). When two springs are put in series (as $K_{1}-K_{2}$ and $K_{1}-K_{3}$ ), the softest one plays the major role. This implies that at the beginning of osseointegration the system can be approximated as shown in Fig. 7c: both cervically and apically the major contribution is given by the maturing bone stiffness $\mathrm{K}_{1}$. As a result, on the left side of Fig. 5, the resonance frequencies are very sensitive to the osseointegration level and show a monotonic increment.

At the end of the osseointegration process the reference model is more complex, being the one shown in Fig.

$7 \mathrm{~d}$. Cervically there are two equivalent stiffness $\left(\mathrm{K}_{2}\right)$, while apically, $\mathrm{K}_{3}$ plays the major role. The respective weight of upper $\left(\mathrm{K}_{1}, \mathrm{~K}_{2}\right)$ and lower $\left(\mathrm{K}_{2}, \mathrm{~K}_{3}\right)$ springs depends on the implant shape (cylindrical or tapered) and on the mode of vibration being analyzed. For example, they have comparable contributions in relation to 
a vertical translation of the implant, while their respective contributions change in relation to the height of the center of rotation when the implant rotates in the mesio-distal or bucco-lingual plane.

When a 'long' bone block is considered (Fig. 3), the first (i.e the lowest frequency) vibration modes are produced by deformation of the mandibular bone itself, with minimum displacement of the implant relative to the bone. Experimental results illustrating such a behavior can be found in the literature. In [23], for example, the mode frequency (around $6 \mathrm{KHz}$ ) did not undergo significant changes as time-from-implantation elapsed for 'successful implants'. So this could indicate that the frequency being measured corresponded to a mandibular bone vibration mode. The predominance of mandibular deformation on implant displacement within bone has been also outlined in numerical work by Natali et al. [19]. These authors suggested the use of a cantilever mass to detect within-bone implant displacements among the first modal shapes. However, this solution has not been implemented in currently used implant devices.

When the 'short' bone block was evaluated, mandibular bone vibration frequencies moved to higher values (the flexural frequency of beams is proportional to the respective squared length) and the first (i.e the lowest frequency) vibration modes were produced by displacements of the implant relative to the bone, provided that bone maturation was less than $20 \%$. The resultant numerical findings can be qualitatively compared to in vitro experimental results obtained by Huang et al. [12], where Brannemark dental implants were implanted into bone blocks, which were cut from the lumbar vertebrae of hogs. The the first vibration frequency was around $10 \mathrm{kHz}$ (for $1 \mathrm{~mm}$ exposed height implants).

An implication of these findings is that the order of vibration modes may be different, depending on the modes of vibration excited in the mandibular bone. Such effects may occur whenever implants are well osseointegrated but the mandible is not supported (unsupported chin; slack muscles) and also the studied implant is distant from the mandibular condyle (that is its main constraint). So the 'first' modes of vibration represent whole mandible vibrations and are less sensitive to osseointegration. Whole mandible modes of vibration can also explain why resonance frequencies are dependent upon different implant locations [20]: the distance of the point of excitation from the boundary constraints determines which mandible vibration modes are actually excited.

Another implication of these findings is to suggest an experimental protocol to verify which kind of modes are actually measured. If the mode frequency undergoes a significant change in relation to boundary 
conditions (supported/unsupported chin; tight/slack muscles), then whole bone vibration modes are likely to be measured, which are less sensitive to osseointegration level.

Different implant shapes and threads can result in different trends of resonance frequency versus osseointegration level. In more detail: tapered implants (' $\mathrm{C}$ ' and ' $\mathrm{D}$ ' models in the present paper) were found to be the most sensitive to osseointegration level.

Modes of vibrations where implant displacement is relevant have proved to be more sensitive to osseointegration, especially when a linear displacement of the implant along the 3 -axis is involved. In vivo experiments on pins inserted in fractured tibia of dogs have shown that the natural frequency did not change even if radiographic changes around the interface were observed [37]. But this could be explained by the reduced size of the implant (see model 'E' results in this study).

As a general rule, modal analysis methods provide a more sensitive estimation of the osseointegration process in the very initial phase, and they give fair indications when the ossointegration level is beyond $50 \%$, or even lower in those areas of the mandible which undergo large displacement. This finding agrees with results obtained by Li and coworkers through a numerical model where also bone remodeling was simulated [18].

Optimization of modal frequency analysis as a clinical tool to evaluate bone integration requires establishment of repeatable and detailed protocols to be able to measure the same mode of vibration. Numerical models like those developed here and in previous work [27-32] can provide a useful tool to understand experimental results and to optimize the measurement protocol. These models can be further improved by simulating bone maturation. A simplified remodeling law has been here assumed where mechanical properties are uniform in the cylinder surrounding the bone implant, but more complex behavior could be simulated, by calculating bone remodeling in relation to bone loads [18], or even by incorporating transport phenomena governing bone metabolism through multiphysics models [38].

\section{Conclusions}

The following conclusions were drawn: 
1. Resonance frequencies and their pattern towards osseointegration level are significantly different between implants having different outer shapes and thread pitches; tapered implants are the most sensitive to bone maturation levels; small implants have very low sensitivity.

2. The trend of resonance frequency versus osseointegration level is similar between flexural modes of vibration on mesio-distal and bucco-lingual planes.

3. Studying axial modes of vibration could bring a limited benefit.

4. Resonance frequencies generally showed reduced sensitivity to bone maturation levels beyond $50 \%$.

\section{References}

[1] Virdi AS, Irish J, Sena K, Liu M, Ke HZ, McNulty MA, et al. Sclerostin Antibody Treatment Improves Implant Fixation in a Model of Severe Osteoporosis. J Bone Jt Surg 2015;97:133-40. doi:10.2106/JBJS.N.00654.

[2] Calì M, Zanetti EM, Oliveri SM, Asero R, Ciaramella S, Martorelli M, et al. Influence of thread shape and inclination on the biomechanical behaviour of plateau implant systems. Dent Mater 2018. doi:10.1016/j.dental.2018.01.012.

[3] Zanetti EM, Aldieri A, Terzini M, Calì M, Franceschini G, Bignardi C. Additively manufactured custom load-bearing implantable devices: Grounds for caution. Australas Med J 2017;10:694-700. doi:10.21767/AMJ.2017.3093.

[4] Heo D, Heo Y-K, Lee J-H, Lee J-J, Kim B. Comparison Between Cortical Drill and Cortical Tap and Their Influence on Primary Stability of Macro-Thread Tapered Implant in Thin Crestal Cortical Bone and Low-Density Bone. Implant Dent 2017;26:711-7. doi:10.1097/ID.0000000000000614.

[5] Brunski JB. Avoid pitfalls of overloading and micromotion of intraosseous implants. Dent Implantol Update 1993;4:77-81.

[6] Duyck J, Vandamme K. The effect of loading on peri-implant bone: a critical review of the literature. J Oral Rehabil 2014;41:783-94. doi:10.1111/joor.12195.

[7] Al-Sawai A-A, Labib H. Success of immediate loading implants compared to conventionally-loaded 
implants: a literature review. J Investig Clin Dent 2016;7:217-24. doi:10.1111/jicd.12152.

[8] Hong DGK, Oh J. Recent advances in dental implants. Maxillofac Plast Reconstr Surg 2017;39:33. doi:10.1186/s40902-017-0132-2.

[9] Schimmel M, Srinivasan M, Herrmann FR, Müller F. Loading protocols for implant-supported overdentures in the edentulous jaw: a systematic review and meta-analysis. Int J Oral Maxillofac Implants 2014;29 Suppl:271-86.

[10] Chung S, McCullagh A, Irinakis T. Immediate Loading in the Maxillary Arch: Evidence-Based Guidelines to Improve Success Rates: A Review. J Oral Implantol 2011;37:610-21. doi:10.1563/AAID-D-JOI-10-00058.1.

[11] Papaspyridakos P, Chen C-J, Chuang S-K, Weber H-P. Implant loading protocols for edentulous patients with fixed prostheses: a systematic review and meta-analysis. Int J Oral Maxillofac Implants 2014;29 Suppl:256-70.

[12] Menicucci G, Ceruti P, Barabino E, Screti A, Bignardi C, Preti G. A preliminary in vivo trial of load transfer in mandibular implant-retained overdentures anchored in 2 different ways: allowing and counteracting free rotation. Int J Prosthodont n.d.;19:574-6.

[13] Huang H-M, Lee S-Y, Yeh C-Y, Lin C-T. Resonance frequency assessment of dental implant stability with various bone qualities: a numerical approach. Clin Oral Implants Res 2002;13:65-74. doi:10.1034/J.1600-0501.2002.130108.X.

[14] Miyamoto I, Tsuboi Y, Wada E, Suwa H, lizuka T. Influence of cortical bone thickness and implant length on implant stability at the time of surgery—clinical, prospective, biomechanical, and imaging study. Bone 2005;37:776-80. doi:10.1016/J.BONE.2005.06.019.

[15] (OFFA) OF for A. Hip Dysplasia Statistics. Hip Dysplasia by Breed and Rank. Trends in Hip Dysplasia (selected breeds) n.d. http://www.offa.org/stats_hip.html?view=2 (accessed May 6, 2016).

[16] Friberg B, Sennerby L, Meredith N, Lekholm U. A comparison between cutting torque and resonance frequency measurements of maxillary implants. Int J Oral Maxillofac Surg 1999;28:297-303. doi:10.1016/S0901-5027(99)80163-5.

[17] Farré-Pagès N, Augé-Castro ML, Alaejos-Algarra F, Mareque-Bueno J, Ferrés-Padró E, HernándezAlfaro F. Relation between bone density and primary implant stability. Med Oral Patol Oral Cir 
Bucal 2011;16:62-7. doi:10.4317/medoral.16.e62.

[18] Li W, Lin D, Rungsiyakull C, Zhou S, Swain M, Li Q. Finite element based bone remodeling and resonance frequency analysis for osseointegration assessment of dental implants. Finite Elem Anal Des 2011;47:898-905. doi:10.1016/j.finel.2011.03.009.

[19] Natali AN, Pavan PG, Schileo E, Williams KR. A numerical approach to resonance frequency analysis for the investigation of oral implant osseointegration. J Oral Rehabil 2006;33:674-81. doi:10.1111/j.1365-2842.2006.01610.x.

[20] Sjöström M, Sennerby L, Nilson H, Lundgren S. Reconstruction of the atrophic edentulous maxilla with free iliac crest grafts and implants: A 3-year report of a prospective clinical study. Clin Implant Dent Relat Res 2007;9:46-59. doi:10.1111/j.1708-8208.2007.00034.x.

[21] Sennerby L, Meredith N. Implant stability measurements using resonance frequency analysis: biological and biomechanical aspects and clinical implications. Periodontol 2000 2008;47:51-66. doi:10.1111/j.1600-0757.2008.00267.x.

[22] Simunek A, Strnad J, Kopecka D, Brazda T, Pilathadka S, Chauhan R, et al. Changes in stability after healing of immediately loaded dental implants. Int J Oral Maxillofac Implants 2010;25:1085-92.

[23] Huwiler MA, Pjetursson BE, Bosshardt DD, Salvi GE, Lang NP. Resonance frequency analysis in relation to jawbone characteristics and during early healing of implant installation. Clin Oral Implants Res 2007;18:275-80. doi:10.1111/j.1600-0501.2007.01336.x.

[24] Glauser R, Sennerby L, Meredith N, Rée A, Lundgren AK, Gottlow J, et al. Resonance frequency analysis of implants subjected to immediate or early functional occlusal loading. Successful vs. failing implants. Clin Oral Implants Res 2004;15:428-34. doi:10.1111/j.1600-0501.2004.01036.x.

[25] Baltayan S, Pi-Anfruns J, Aghaloo T, Moy PK. The predictive value of resonance frequency analysis measurements in the surgical placement and loading of endosseous implants. J Oral Maxillofac Surg 2016;74:1145-52. doi:10.1016/j.joms.2016.01.048.

[26] Nedir R, Bischof M, Szmukler-Moncler S, Bernard JP, Samson J. Predicting osseointegration by means of implant primary stability: A resonance-frequency analysis study with delayed and immediately loaded ITI SLA implants. Clin Oral Implants Res 2004;15:520-8. doi:10.1111/j.16000501.2004.01059.x. 
[27] Giordano M, Ausiello P, Martorelli M. Accuracy evaluation of surgical guides in implant dentistry by non-contact reverse engineering techniques. Dent Mater 2012;28:e178-85. doi:10.1016/j.dental.2012.06.006.

[28] Ausiello P, Ciaramella S, Garcia-Godoy F, Gloria A, Lanzotti A, Maietta S, et al. The effects of cavity-margin-angles and bolus stiffness on the mechanical behavior of indirect resin composite class II restorations. Dent Mater 2017;33:e39-47. doi:10.1016/j.dental.2016.11.002.

[29] Ausiello P, Ciaramella S, Fabianelli A, Gloria A, Martorelli M, Lanzotti A, et al. Mechanical behavior of bulk direct composite versus block composite and lithium disilicate indirect Class II restorations by CAD-FEM modeling. Dent Mater 2017;33:690-701. doi:10.1016/j.dental.2017.03.014.

[30] Ausiello P, Ciaramella S, Garcia-Godoy F, Martorelli M, Sorrentino R, Gloria A. Stress distribution of bulk-fill resin composite in class II restorations. Am J Dent 2017;30:227-32.

[31] Ausiello P, Ciaramella S, Martorelli M, Lanzotti A, Gloria A, Watts DC. CAD-FE modeling and analysis of class II restorations incorporating resin-composite, glass ionomer and glass ceramic materials. Dent Mater 2017;33:1456-65. doi:10.1016/j.dental.2017.10.010.

[32] Ausiello P, Ciaramella S, Martorelli M, Lanzotti A, Zarone F, Watts DC, et al. Mechanical behavior of endodontically restored canine teeth: Effects of ferrule, post material and shape. Dent Mater 2017;33:1466-72. doi:10.1016/j.dental.2017.10.009.

[33] Calì M, Oliveri SM, Fatuzzo G, Sequenzia G. Error control in UAV image acquisitions for 3D reconstruction of extensive architectures, Springer, Cham; 2017, p. 1209-19. doi:10.1007/978-3-31945781-9_121.

[34] Carter DR, Hayes WC. The Compressive Behavior Porous of Bone Structure as a Two-Phase Porous Structure. J Bone Jt Surg 1977;59:954-62. doi:10.1007/978-1-4471-5451-8_116.

[35] Calì M., Oliveri S. M., Ambu R., \& Fichera G. (2018). An Integrated Approach to Characterize the Dynamic Behaviour of a Mechanical Chain Tensioner by Functional Tolerancing. Strojniski Vestnik/Journal of Mechanical Engineering, 64(4). doi:10.5545/sv-jme.2017.5079

[36] Calì M, Oliveri SM, Sequenzia G, Fatuzzo G. An effective model for the sliding contact forces in a multibody environment. Lect Notes Mech Eng 2017:675-85. doi:10.1007/978-3-319-45781-9_68. 
[37] Kay MW, Roe SC, Stikeleather LF, Mahmoud A, Abrams CF. Axial vibration of threaded external fixation pins: detection of pin loosening. Ann Biomed Eng n.d.;26:361-8.

[38] De Napoli IE, Zanetti EM, Fragomeni G, Giuzio E, Audenino AL, Catapano G. Transport modeling of convection-enhanced hollow fiber membrane bioreactors for therapeutic applications. J Memb Sci 2014;471:347-61. doi:10.1016/j.memsci.2014.08.026. 
Figures and figure captions

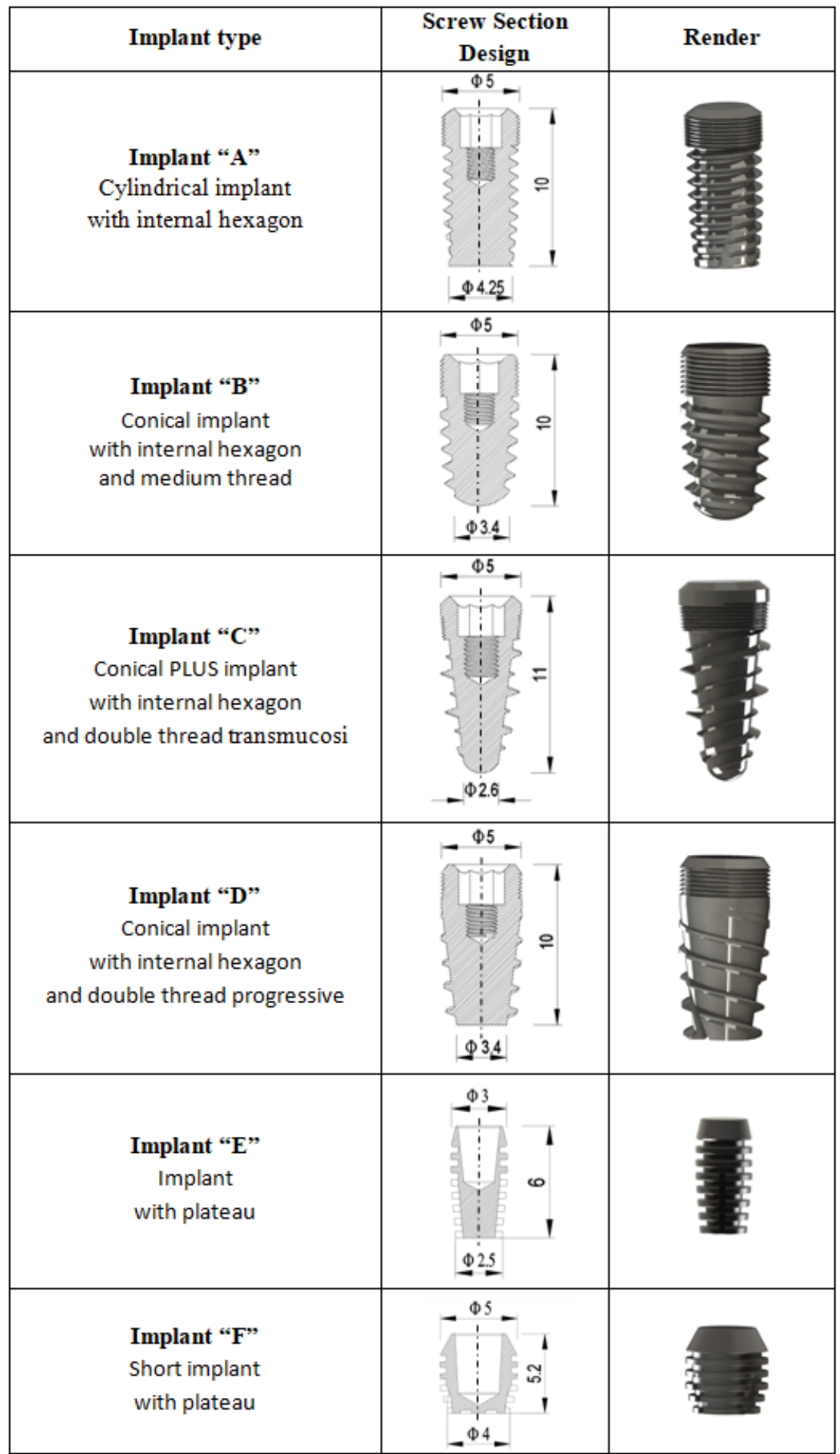

Fig. 1 - ISOMED implants: geometric characteristics. 


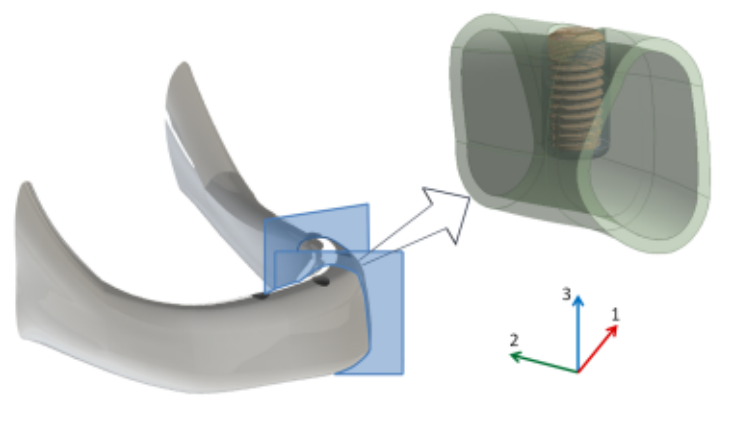

a)

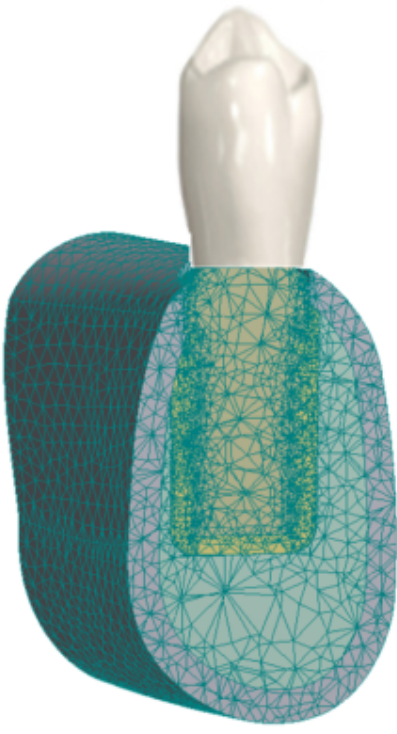

b)

Fig. 2 - (a) Bone-implant system: (a) geometry and coordinate system; (b) FE model. 

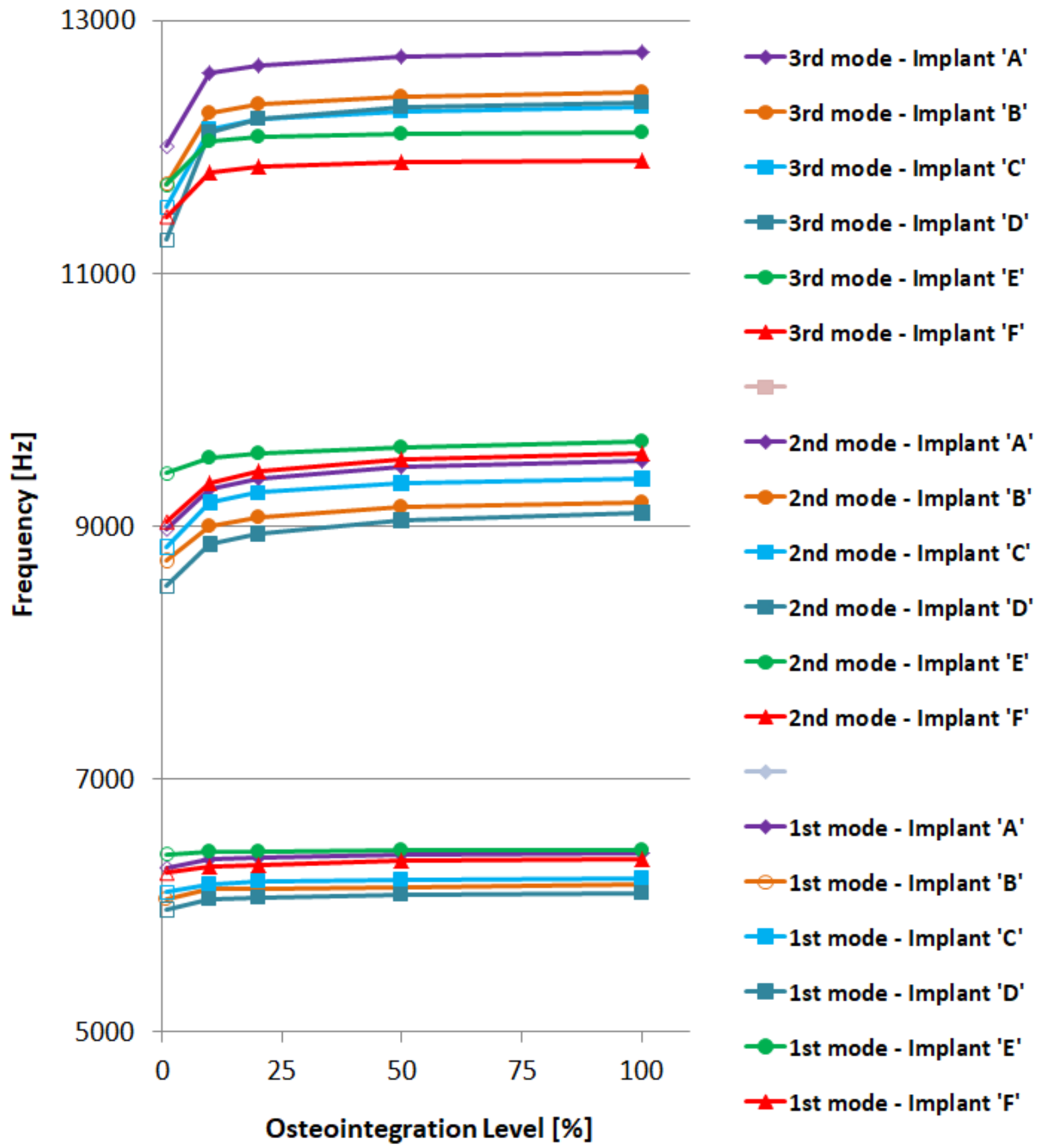

Fig. 3 - Modal frequencies versus osseointegration level for different implant designs for the first boundary condition ('long' mandibular branch); hollow markers refer to modes where the movement of the implant inside bone is evident 

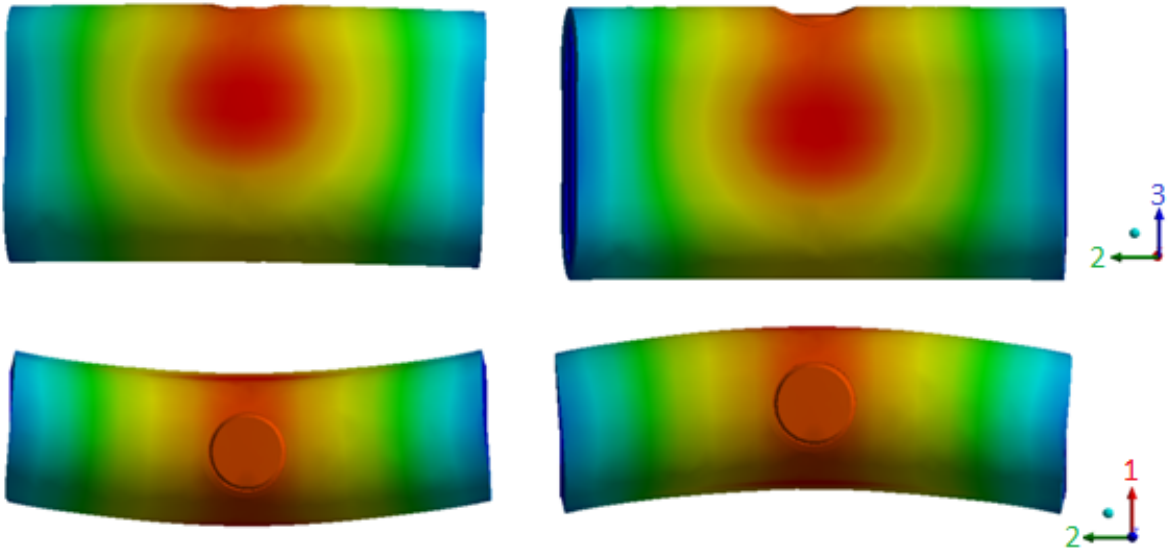

a)
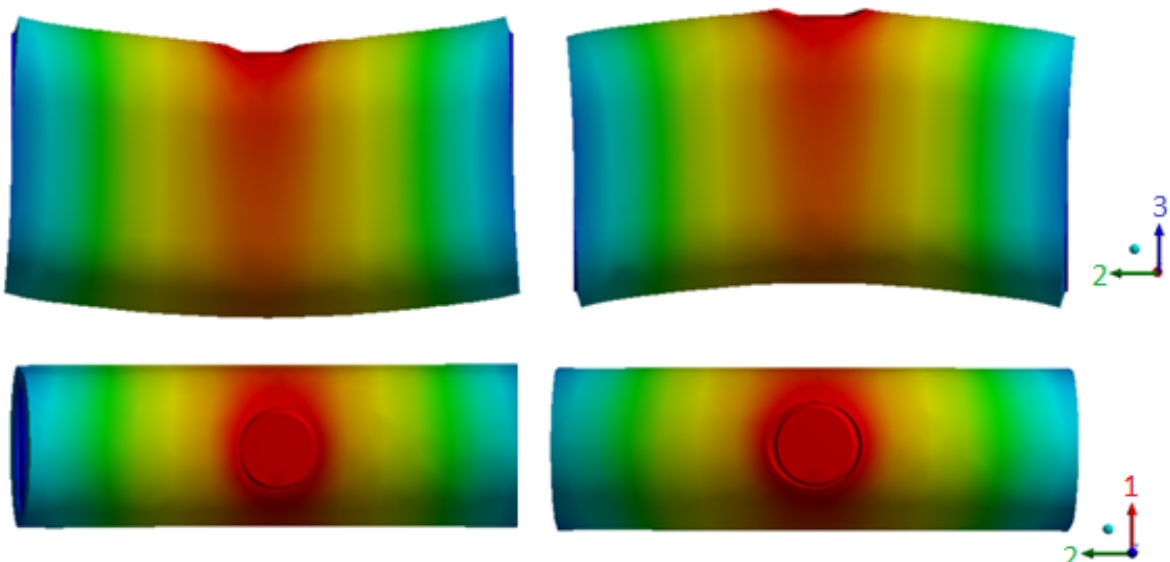

b)
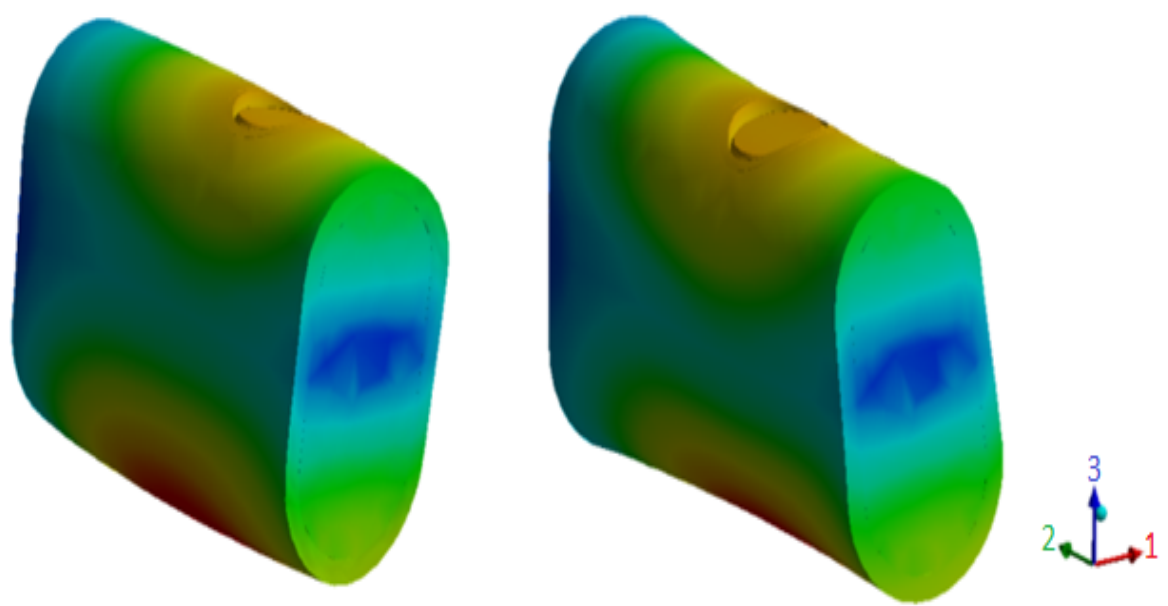

c)

Fig. 4 - Modal shapes for the 'long' mandibular branch, constrained at its ends and k>0.1: (a) $1^{\text {st }}$ mode (flexion about ' 3 ' axis); (b) $2^{\text {rd }}$ mode (flexion about ' 1 ' axis); (c) $3^{\text {rd }}$ mode (torsion) 


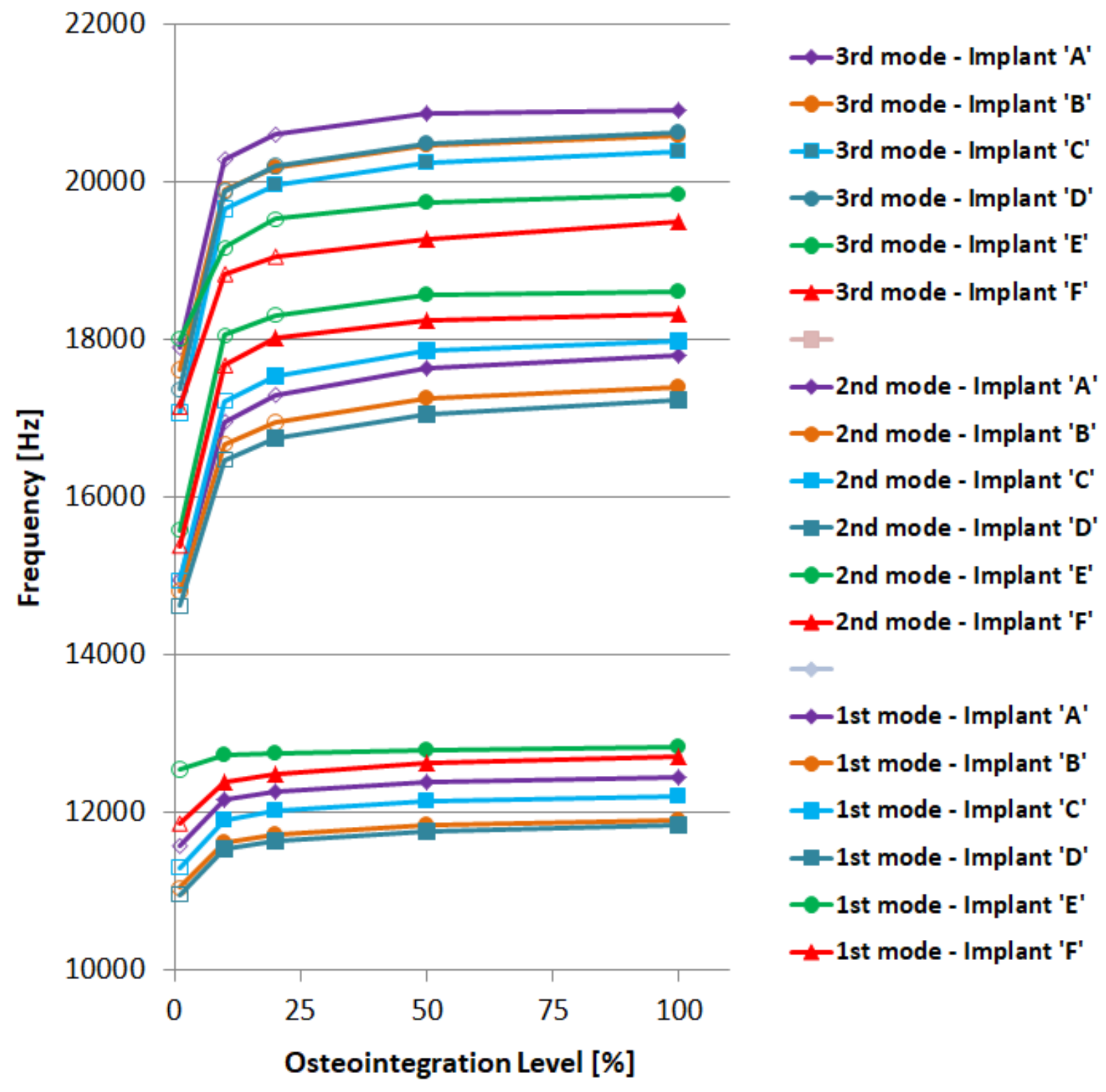

Fig. 5 - Modal frequencies versus osseointegration level for different implant designs for the second boundary condition ('short' mandibular branch); hollow markers refer to modes where the movement of the implant inside bone is evident 

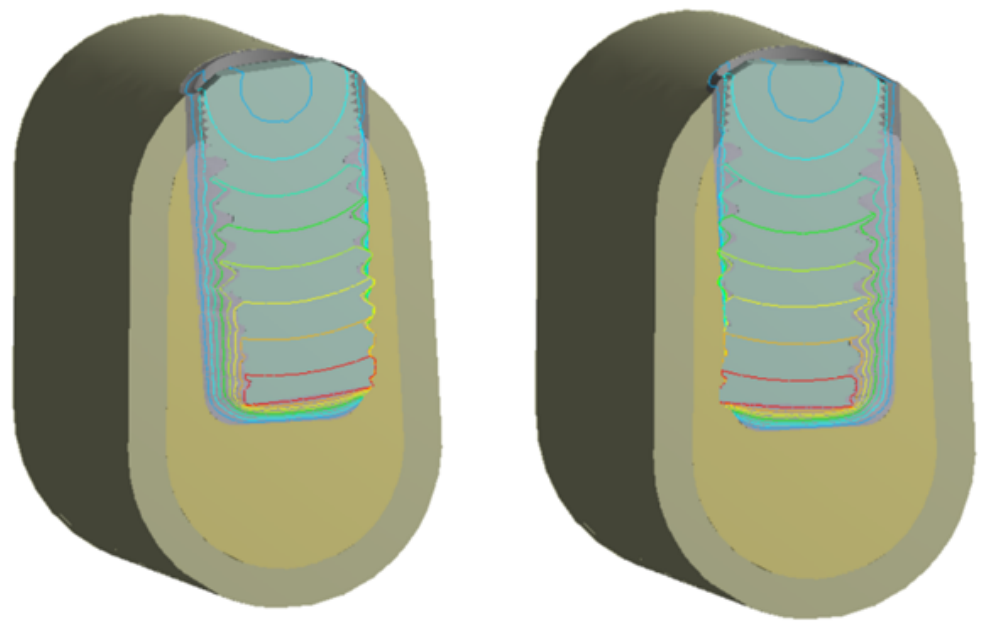

a)
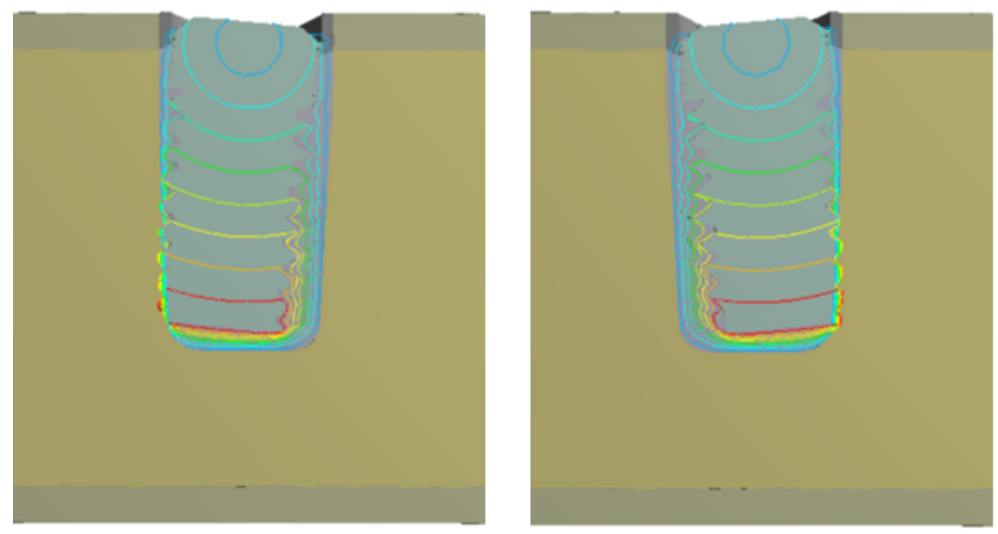

b)
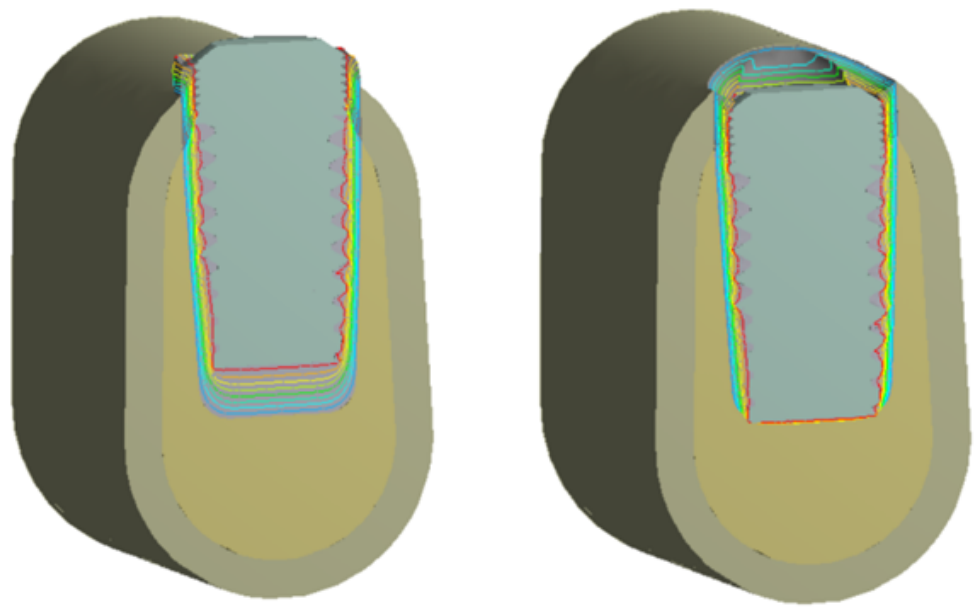

c)

Fig. 6 - Modal shapes for the 'short' mandibular branch: (a) $1^{\text {st }}$ mode for $k=0.01$ (rotation about '2' axis); (b) $2^{\text {nd }}$ mode for $k<0.5$ (rotation about ' 1 ' axis); (c) $3^{\text {rd }}$ mode for $k<0.5$ (axial displacement). 


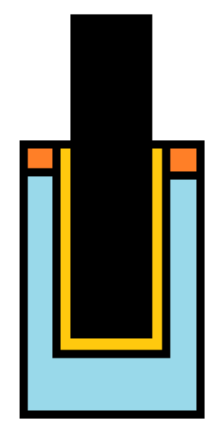

a)

b)

c)

d)

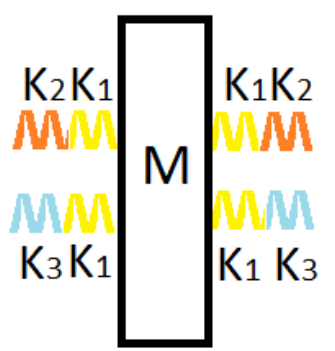

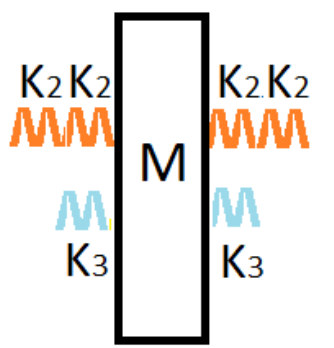

Fig. 7 - Simplified dynamic model of bone implant system: physical model (a); equivalent dynamic model (b); immediate post-operative condition (c); long-term result (d) 
Table and caption

\begin{tabular}{|c|c|c|c|c|c|}
\hline & \multirow[t]{2}{*}{$\begin{array}{c}\text { Implant } \\
\text { (titanium) }\end{array}$} & \multicolumn{2}{|c|}{ Trabecular Bone } & \multicolumn{2}{|c|}{ Cortical Bone } \\
\hline & & $\begin{array}{l}\text { Massive } \\
\text { Volume }\end{array}$ & $\begin{array}{c}\text { Interface } \\
\text { Volume }\end{array}$ & $\begin{array}{l}\text { Massive } \\
\text { Volume }\end{array}$ & Interface Volume \\
\hline$\rho\left[\mathrm{Kg} / \mathrm{m}^{3}\right]$ & 4620 & 500 & $500 \cdot \mathrm{k}^{1 / 3 *}$ & 1800 & $1800 \cdot \mathrm{k}^{1 / 3 *}$ \\
\hline $\mathrm{E}_{1}[\mathrm{MPa}]$ & 120000 & 500 & $500 \cdot \mathrm{k}^{*}$ & 10000 & $10000 \cdot \mathrm{k}^{*}$ \\
\hline $\mathbf{E}_{2}[\mathbf{M P a}]$ & 120000 & 1000 & $1000 \cdot \mathrm{k}^{*}$ & 13000 & $13000 \cdot \mathrm{k}^{*}$ \\
\hline $\mathrm{E}_{3}[\mathrm{MPa}]$ & 120000 & 500 & $500 \cdot \mathrm{k}^{*}$ & 10000 & $10000 \cdot \mathrm{k}^{*}$ \\
\hline$v_{1}$ & 0.33 & 0.222 & 0.222 & 0.3 & 0.3 \\
\hline$v_{2}$ & 0.33 & 0.246 & 0.246 & 0.3 & 0.3 \\
\hline$v_{3}$ & 0.33 & 0.222 & 0.222 & 0.3 & 0.3 \\
\hline
\end{tabular}

$* k$ parameter defines different levels of osseointegration (the following values have been tested: $k=0.01 ; k=0.1 ; k=0.5 ; k=1$ ).

Table 1 - Mechanical properties of materials. 